\title{
La médecine tous azimuts: problèmes éthiques de la médicalisation du quotidien
}

Notre compréhension de la «maladie» et de la «santé» diverge selon les contextes temporels et culturels cette affirmation n'est guère contestée. Cependant, les implications à la fois sociales et individuelles de la classification des états psychiques et physiques en

\begin{tabular}{|c|c|c|c|}
\hline \multicolumn{2}{|c|}{ Programme } & \multirow{2}{*}{\multicolumn{2}{|c|}{$\begin{array}{l}\text { Problèmes éthiques de la médicalisation du } \\
\text { quotidien - aspects systématiques }\end{array}$}} \\
\hline \multirow{6}{*}{$9 \mathrm{~h} 15$} & Allocution de bienvenue et & & \\
\hline & introduction & $13 \mathrm{~h} 30$ & Medikalisierung des Alltags: \\
\hline & Prof. Otfried Höffe, Präsident & & Anreize und Kosten \\
\hline & der NEK-CNE, Tübingen & & Prof. Stefan Felder, \\
\hline & Prof. Christian Kind, Präsident & & Universität Basel \\
\hline & $\begin{array}{l}\text { der Zentralen Ethikkommission } \\
\text { der SAMW, St. Gallen }\end{array}$ & $13 \mathrm{~h} 50$ & $\begin{array}{l}\text { Gesellschaftliche Hintergründe } \\
\text { der Medikalisierung }\end{array}$ \\
\hline & & & Prof. Peter C. Meyer, Zürcher \\
\hline \multicolumn{2}{|c|}{ Exemples pratiques } & & Hochschule für Angewandte \\
\hline \multirow[t]{6}{*}{ 9h35 } & $\begin{array}{l}\text { Le syndrome de carence en fer- } \\
\text { une maladie nouvelle? }\end{array}$ & 14h10 & Wissenschaften, Winterthur \\
\hline & Dr. Roch Ogier, MD-PhD, & 141110 & rung \\
\hline & medical Director Vifor, Genève & & Prof. Johannes Fischer, \\
\hline & Dr. Franziska Demarmels & & Universität Zürich \\
\hline & Biasiutti, Stellvertretende & $14 \mathrm{~h} 30$ & Discussion \\
\hline & $\begin{array}{l}\text { Chefärztin Hämatologie, } \\
\text { Insel-Spital Bern }\end{array}$ & $15 \mathrm{~h} 15$ & Pause \\
\hline \multirow[t]{8}{*}{ 10h05 } & $\begin{array}{l}\text { Le burn-out: diagnostic ou } \\
\text { lifestyle? }\end{array}$ & $15 \mathrm{~h} 45$ & Table ronde: L'économicisation \\
\hline & $\begin{array}{l}\text { PD Dr. Peter Schneider, } \\
\text { Psychoanalytiker und }\end{array}$ & & $\begin{array}{l}\text { de la médecine: symptôme ou } \\
\text { cause de la médicalisation? }\end{array}$ \\
\hline & Kolumnist, Zürich & & Yves Rossier, Directeur, Office \\
\hline & PD Dr. Alessandra Canuto, & & $\begin{array}{l}\text { fédéral des assurances sociales, } \\
\text { Berne }\end{array}$ \\
\hline & Cheffe de service a.i., Servi & & PD Dr. Iris Ritzmann, \\
\hline & d'intervention de crise. & & Universität Zürich \\
\hline & Hôpitaux Universitaires de & & Dr. Constantin Schuler, \\
\hline & Genève & & Spezialarzt für Orthopädische \\
\hline \multirow[t]{2}{*}{$10 \mathrm{~h} 35$} & Discussion & & Chirurgie, Mörschwil \\
\hline & & & Prof. Bernice Elger,Université \\
\hline \multirow[t]{2}{*}{ 11h00 } & Pause & & \\
\hline & & & Dr. Dirk Lanzerath, DRZE, Bonn \\
\hline \multicolumn{2}{|c|}{ Santé et maladie: aspects conceptuels } & & Moderation: Prof. Iwan \\
\hline \multirow[t]{2}{*}{$11 \mathrm{~h} 30$} & $\begin{array}{l}\text { Krankheitsbilder als Spiegel } \\
\text { ihrer Zeit }\end{array}$ & & Rickenbacher, Schwyz \\
\hline & $\begin{array}{l}\text { PD Dr. Iris Ritzmann, } \\
\text { Universität Zürich }\end{array}$ & $17 \mathrm{~h} 00$ & $\begin{array}{l}\text { Synthèse et perspectives } \\
\text { Prof. Otfried Höffe, Präsident }\end{array}$ \\
\hline \multirow[t]{4}{*}{$11 \mathrm{~h} 50$} & Gesundheit und Krankheit als & & der NEK-CNE, Tübingen \\
\hline & normative Begriffe & & Prof. Christian Kind, Präsident \\
\hline & Dr. Dirk Lanzerath, Deutsches & & der Zentralen Ethikkommission \\
\hline & Referenzzentrum für Ethik in & & der SAMW, St. Gallen \\
\hline $12 \mathrm{~h} 10$ & $\begin{array}{l}\text { den Biowissenschatten, Bonn } \\
\text { Discussion }\end{array}$ & $17 \mathrm{~h} 15$ & Fin de la journée \\
\hline $12 \mathrm{~h} 30$ & Lunch & Tradu & simultanée allemand-français \\
\hline
\end{tabular}

«maladie» ou en «santé» ne sont devenues que récemment un sujet de réflexion. Car nous vivons sans nul doute dans une société qui définit - souvent sur la base de raisonnements médicaux - toujours davantage de phénomènes jugés indésirables au plan social ou individuel en termes de maladie et les fait accompagner par des traitements médicaux spécifiques. Ce processus, qui englobe à la fois des diagnostics psychiques et somatiques et qui touche souvent notre quotidien au sens large, est désigné par le terme de médicalisation.

Basé sur les deux exemples pratiques du «syndrome de carence en fer» et du «burn-out» ainsi que sur une analyse historique, le symposium de la Commission nationale d'éthique pour la médecine humaine (NEK-CNE) et de l'Academie suisse des sciences médicales (ASSM) entend engager un débat critique de cette médicalisation du quotidien. Car s'il est vrai que le fait de pouvoir associer son état à un diagnostic particulier peut représenter, d'une part, un soulagement pour la personne concernée, la médicalisation croissante peut également induire, d'autre part, une perte d'autonomie et des pressions sociales accrues. Ceci par exemple par rapport au choix de recourir ou non à des traitements médicaux ou alors en vue du financement solidaire des thérapies.

Le symposium sera consacré aux aspects sociologiques et éthiques de la médicalisation, mais abordera aussi les questions économiques soulevées par ce processus, questions qui sont quant à elles d'une grande importance éthique. Car la médicalisation du quotidien ouvre bien des perspectives de commercialisation, mais ne va pas sans générer des coûts, tant pour les patients que pour la collectivité. Ainsi, les incitatifs encourageant la médicalisation du quotidien et les coûts qui en découlent seront discutés et examinés. Par rapport au thème général du cycle de conférences de la NEK-CNE et de l'ASSM, la question de l'interdépendance entre l'influence croissante des facteurs économiques en médecine et la médicalisation du quotidien sera débattue.

Informations et inscriptions: www.assm.ch 